\title{
Evoked responses to retroactively masked stimuli
}

\author{
MARVIN SCHWARTZ, ONCY M. WHITTIER \\ University of Cincinnati, Cincinnati, Ohio 45221 \\ and \\ PAULA K. SCHWEITZER \\ Xavier University, Cincinnati, Ohio 45207
}

\begin{abstract}
Recordings of evoked responses in three backward masking demonstrations indicated that during perceptual masking of a discrimination a residual representation of the targets remains in the nervous system. In a procedure employing two masks, recovery of target discrimination occurred but the residual response to the targets remain degraded. These results imply that target discrimination depends on some minimal portion of the total evoked response to the targets.
\end{abstract}

In backward masking, two stimuli are presented in close temporal continguity; with appropriate adjustment of the interval between the two stimuli, the second stimulus (mask) may interfere with perception of the first (target). Thus, a target which is normally detected or discriminated when presented alone, may not be, or it may be altered in appearance, when followed by a second stimulus. There have been several prior averaged evoked response (AER) studies of masked targets, but some questions are unanswered or ambiguously answered. Studies in humans (Donchin \& Lindsley, 1965; Donchin, Wicke, \& Lindsley, 1963) generally suggest that AERs to targets are "erased" or displaced by those to the mask. But since perception of masked targets can be recovered if a second mask is employed-the second mask masks the first one-neural responses to masked targets cannot have been permanently displaced (Dember \& Purcell, 1967; Dember, Schwartz, \& Kocak, 1978; Robinson, 1966). Studies in the cat and monkey (Coenen \& Eijkman, 1972; Fehmi, Adkins, \& Lindsley, 1969; Schiller, 1969) suggest that masking results from failure of neural units to respond differentially to the combination of target and mask vs. mask alone. They also suggest that processes in the retina or lateral geniculate account for masking. But since dichoptic stimulating conditions can produce masking (e.g., Turvey,

This study was supported by a grant from the University of Cincinnati Research Council to M.S. We thank Donna Arand, Robert Waite, Richard Young, and Robert Lysaght for the considerable time they devoted to serving as subjects and helping to collect portions of these data. William N. Dember and Joel S. Warm gradiously provided advice and constructive criticism. Address reprint requests to Marvin Schwartz, Provost's Office, 101 Administration Building, University of Cincinnati, Cincinnati, Ohio 45221.
1973), these loci cannot be solely responsible for all masking effects.

The experiments presented here are demonstrations. Their major intent is to show that (1) for a variety of stimulus conditions employing discrimination criteria as the measure of masking, perceptually masked stimuli have a residual representation in the nervous system, and (2) this degraded residual representation is not altered under conditions resulting in recovery of the discrimination.

\section{GENERAL METHODS}

All subjects were generally familiar with backward masking and AER techniques. With their heads stabilized, they viewed the stimuli through 4-mm appertures in an Iconix three-field tachistoscope. The stimuli were backlighted through holes in an opaque background. Four dim red spots of light in a $\mathrm{Y}$ shape provided a fixation figure. EEG recordings were obtained using silver or gold electrodes, filled with electrode jelly and attached to the scalp with collodion according to the International 10-20 system. Similar electrodes were used to monitor eye movements. Recordings were amplified by a Grass Model 7 polygraph and stored on FM tape for processing with an Enhancetron averager. The recording system had a frequency range of $.3-1,250 \mathrm{~Hz}$. Subjects self-initiated trials after fixating and receiving verbal indication of no eye movement or artifacts. Trials were at least $7 \mathrm{sec}$ apart, in blocks, with 5-10 min between blocks. Eyeblinks and report of observations were delayed until $1 \mathrm{sec}$ or more after a stimulus trial. No feedback was given for subjects' responses. Recording sessions were a maximum of $3 \mathrm{~h}$ long.

From AERs produced by a combination of targets and masks, the averager subtracted the AER produced by masks presented alone (at the time after a target would have been presented). This procedure can only provide a crude estimate of any residual response to the target, since the waveform of the AER to the mask presented alone is not likely to be identical to the waveform of the mask preceded by a target. But for this reason, our procedure does test the conclusion that targets are erased or displaced by the mask (Donchin et al., 1963; Donchin \& Lindsley, 1965). In other words, any residual does indicate that the target 
was not simply eliminated. This analysis is patterned after studies of AER recovery cycles (e.g., Schwartz \& Shagass, 1964), and similar procedures have been used in masking studies (Donchin \& Lindsley, 1965; Donchin, Wicke, \& Lindsley, 1963; Schiller \& Chorover, 1966; Vaughan \& Silverstein, 1968).

\section{EXPERIMENT 1}

To separate retinal and central influences in masking, comparisons between monoptic and dichoptic stimulating conditions are necessary; so far as we are aware, no dichoptic AER studies have been reported. Such comparisons should employ parafoveal presentations to ensure that in dichoptic conditions central interactions of stimuli result from the projection of the stimuli to the same hemisphere. But previous studies with parafoveal stimuli suggest that they result in AERs that are unaltered despite perceptual masking of the targets (Schiller \& Chorover, 1966); stray light may be a factor in this result (Vaughan \& Silverstein, 1968). Experiment 1, then, simply tested to see if we could produce AER alterations as a result of masking with parafoveal stimulating conditions.

\section{Method}

Stimuli. Targets were arrowhead-shaped spots of light, $1^{\circ}$ in maximum extent, $6.5 \mathrm{fL}$ in intensity, presented for 3-10 msec in different subjects. The subjects discriminated their up or down orientation. The mask was a concentric annulus with a strip width of $10^{\prime}$ and an inner diameter of $1^{\circ}$. It was presented for $100 \mathrm{msec}$ and was $10 \mathrm{fL}$ in intensity. In different sessions, the interstimulus interval (ISI) between targets and mask was varied between 7 and $50 \mathrm{msec}$. Stimuli were presented $3.5^{\circ}$ into the right visual field in the right eye.

Recordings. EEGs were recorded from bilateral, bipolar occipital-parietal placements $\left(\mathrm{O}_{1}-\mathrm{P}_{3}\right.$ and $\left.\mathrm{O}_{2}-\mathrm{P}_{4}\right)$.

Procedure. In each session, the four subjects first viewed randomly alternated targets in a block of 100 trials. Subsequently, two blocks of 100 trials of masks alone or targets followed by masks were presented; the order of these trial blocks was randomized for each subject and they were not told which was to occur. In all blocks, the subjects' task was to discriminate the orientation of the arrows. Some subjects, in some ISI conditions, could detect whether targets were actually present even when their target discrimination remained poor.

\section{Results and Discussion}

In all subjects, AERs showed shorter latencies for onset and component peaks on the left side of the head (7-10-msec difference). This suggests isolation of the stimuli to the nasal retina, projection to the left hemisphere, and transcallosal conduction to the right hemisphere. If stray light were a factor, it seems unlikely that such latency differences could be recorded.

In all four subjects, targets presented alone were discriminated with $100 \%$ accuracy. With target-mask ISI ranging from 7 to $15 \mathrm{msec}$, target discrimination accuracy fell to $55 \%$ or less in all subjects.

The AERs accompanying this discrimination performance suggested that even during chance discri-
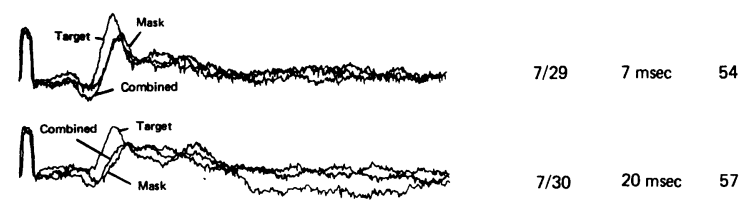

$7 / 30 \quad 20 \mathrm{msec} \quad 57$

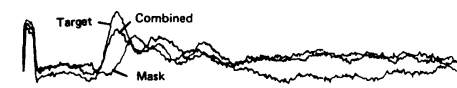

$30 \mathrm{msec}$

65

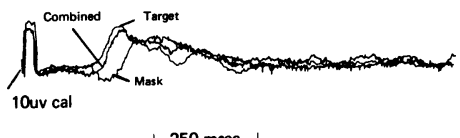

$8 / 4$

$35 \mathrm{msec} \quad 81$

Figure 1. AERs to target stimuli alone, mask alone, and targets and mask together at the indicated ISIs for one subject. Monoptic, parafoveal presentation of stimuli. The combined presentation of targets and mask resulted in AERs that fell between the waveforms of the AERs to the separate stimuli. Occipital negativity up in this and all other figures.

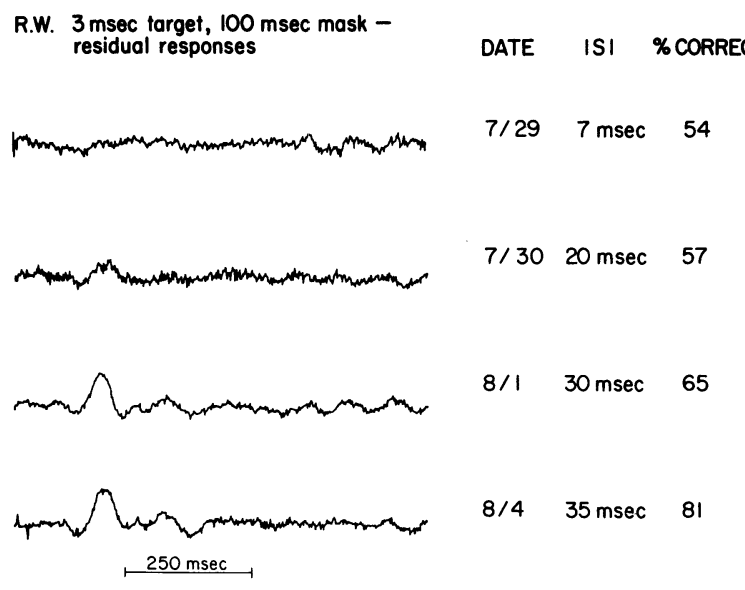

Figure 2. Residual AERs obtained by subtracting the AER to mask stimuli alone from the AER to the combination of targets and mask. These residuals were obtained from the data of Figure 1. Note the presence of at least a minimal residual in all instances. This subject showed the smallest residual of all subjects at short ISIs.

mination the AER to the combination of targets and mask resulted from an interaction of AERs to the separate stimuli. Figure 1 illustrates this for the subject with the largest and clearest AERs-he is also the subject requiring the shortest ISI in order to successfully mask the targets. Three AERs-one to targets alone, one to targets and masks together, and one to masks alone- are overlapped for comparison. Generally, the response to the combination of targets and mask falls between the other two AERs, suggesting there are residual responses to the targets. Our linear subtraction procedure provides an estimate of these residuals; Figure 2 shows such residuals for the four ISIs employed with this subject. During chance discrimination, this subject 


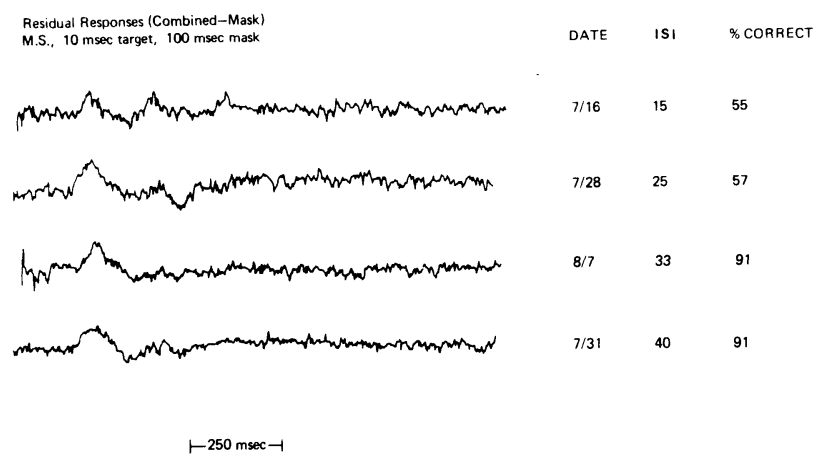

Figure 3. Residual AERs from monoptic parafoveal conditions for another subject. Note the relatively flat gradient of increase in residual as ISI increased.

showed the smallest residual of all four subjects. Figure 3 illustrates the residuals obtained from another subject. These are much larger and show a flatter gradient with ISI. Across the four subjects, during chance discrimination the residuals ranged from $20 \%$ to $80 \%$ smaller than AERs to targets presented alone.

Generally, then, these data illustrate that AER changes during backward masking can be demonstrated with parafoveally presented stimuli. They also suggest that even during chance discrimination it is possible to demonstrate some neural residual of perceptually masked targets.

\section{EXPERIMENT 2}

Experiment 1 generally supported the feasibility of employing parafoveal conditions to investigate AER changes in backward masking. Experiment 2 used dichoptic parafoveal conditions to eliminate retinal interactions between targets and masks.

\section{Method}

Subjects. There were three subjects, two of whom had served in Experiment 1.

Stimuli. Dichoptically presented, the stimuli of Experiment 1 do not produce masking; therefore, patterned stimuli were used here (Turvey, 1973). Targets were black M or W shapes or upright or inverted $\mathrm{V}$ shapes, with a $7^{\prime}$ strip width, on an illuminated background, chosen so as to achieve $100 \%$ discriminability by individual subjects without the mask. The letters were about $30^{\prime}$ in extent; including their background, the targets subtended $40^{\prime}$ and were $10 \mathrm{msec}$ in duration. The mask was randomly placed black bars with a strip width of $7^{\prime}$, on an illuminated background, overlapping the targets. It was $1^{\circ} 20^{\prime}$ in extent, including the background, and $100 \mathrm{msec}$ in duration. The stimuli were presented $3^{\circ} 20^{\prime}$ parafoveally, with targets presented to the temporal retina of the left eye and the mask presented to the nasal retina of the right eye. Polarizing filters placed in front of the stimuli and each eye isolated their presentation. Measured through the filters, targets were $8 \mathrm{fL}$ and the mask was $4 \mathrm{fL}$ in intensity.

Recordings. In all subjects, a variety of monopolar and bipolar electrode arrays were employed to see if AER changes during masking might behave differentially in different scalp locations. Recordings included temporal, central, and frontal areas as well as occipital and parietal locations. In no case was there any indication of effects different from those obtained with bipolar $\mathrm{O}_{1}$ $\mathrm{P}_{3}$ leads which are illustrated here.

Procedure. Targets and mask were viewed alone and in combination in separate 100-trial blocks, and subjects attempted to report target orientation on each trial. Block order was randomized for each subject, and they were uninformed about the nature of each block. ISI was always $10 \mathrm{msec}$ and several sessions were conducted with each subject.

\section{Results and Discussion}

Two subjects consistently showed only chance discrimination of masked targets; the other subject varied between $61 \%$ and $78 \%$ correct in different sessions. However, all three subjects showed substantial target residuals during masking. Figure 4 illustrates the data for the subject of Experiment 1 whose data were shown in Figures 1 and 2. As can be seen, here this subject demonstrated an AER residual that is nearly as large as the AER to the unmasked targets despite the fact that he was discriminating the targets at chance levels. In contrast, under comparable performance conditions in Experiment 1, the residual was minimal in size. The other subject who also participated in Experiment 1 showed residuals here that were twice the size of those of Experiment 1. These results suggest that most of the loss of amplitude of the residuals in monoptic conditions is due to retiral interactions between the stimuli. This inference must be made cautiously, since the stimuli in these two experiments differed physically as well as in the monopticdichoptic dimension. At the least, however, these data indicate that residual responses can be quite large, even though the subject cannot correctly discriminate the targets. In other words, these data demonstrate that, at least with discrimination criteria of masking, responses to the target are not eliminated during perceptual masking.

R.W. DICHOPTIC, $10 \mathrm{msec}$ TARGET, $100 \mathrm{msec}$ MASK

$9 / 76$ $10 \mathrm{msec}$ ISI, $44 \%$ CORRECT

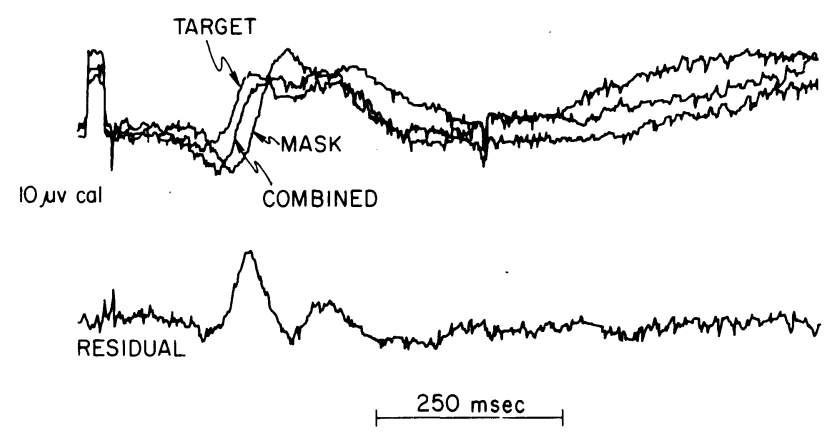

Figure 4. AERs from dichoptic conditions for the same subject whose data were shown in Figures 1 and 2. Despite chance discrimination of the targets, there was a large residual response. At a comparable performance level in monoptic conditions (Figure 2, 54\% correct), he showed an extremely small residual. 


\section{EXPERIMENT 3}

This experiment employed the finding that backward masking effects can be reduced if a second, additional mask is introduced following the original mask (Dember \& Purcell, 1967; Robinson, 1966). Target residuals were compared in single- and doublemask situations in the same subjects. In order to increase the sensitivity of the experiment, we made two changes from prior procedures: We increased the number of alternative targets from two to four, and in the same blocks of trials we included mask-alone trials along with trials combining targets and masks. The latter was designed to ensure the similarity of the tasks required of the subjects in the two different types of trials.

\section{Method}

Subjects. Three subjects were tested, one of whom served in the prior two experiments.

Stimuli. In preliminary work, we attempted to use the stimuli and conditions of Experiment 2. This did not prove feasible. As we quickly discovered with foveal presentation of the stimuli, targets which once again became discriminable, owing to the second mask, appeared dark gray on a grayish background, rather than black on a white background. Thus, our parafoveal conditions either did not provide sufficient acuity to discriminate recovered targets or such recovery did not occur. In either event, our interest was in achieving psychophysical recovery of previously masked targets; for this purpose, foveal stimulation is quite appropriate and, therefore, was employed.

The targets consisted of the four black letters, b, p, d, and q, which appeared on an illuminated circular background, $40^{\prime}$ in diameter. Each letter was $29^{\prime}$ in height, $22^{\prime}$ in width, with a strip width of $7^{\prime}$. Mask 1 was the random bars of Experiment 2, 40' in diameter. Mask 2 was a homogeneous white disk, $1^{\circ} 20^{\prime}$ in diameter. Targets were presented for $7 \mathrm{msec}$ at an intensity of $7 \mathrm{fL}$. Mask 1 was presented for $20 \mathrm{msec}$ at an intensity of $2.5 \mathrm{fL}$. Mask 2 was presented for $50 \mathrm{msec}$ at an intensity of $15 \mathrm{fL}$. ISI between targets and Mask 1 was constant at $10 \mathrm{msec}$ and ISI between Mask 1 and Mask 2 was also a constant $10 \mathrm{msec}$. All stimuli were presented foveally to the right eye, within the area encompassed by the fixation figure.

Recordings.EEGs were 1 ecorded with bipolar $\mathrm{O}_{7}-\mathrm{P}_{7}$ leads.

Procedure. The initial block of trials consisted of 75 randomly selected presentations of the letter targets alone. Discrimination was $100 \%$ accurate in all subjects. Next were three 50 -trial blocks with rests between blocks. Within each block, half the trials consisted of targets followed by Mask 1 and half consisted of Mask 1 alone, randomly alternated. The final set of three blocks randomly alternated trials with (a) targets, Mask 1, and Mask 2, and (b) Mask 1 and Mask 2 alone. On each trial, subjects were required to indicate if a target was present and, if so, which it was.

\section{Results and Discussion}

In the single-mask condition, one subject could discriminate trials with and without targets but could not discriminate among the targets. In the dual mask condition, he correctly discriminated $80 \%$ of the targets.

The second subject could not discriminate trials with or without targets in the single-mask condition; over both types of trials together, he showed $32 \%$ accuracy. In the dual-mask condition, he showed $82 \%$ accuracy across both types of trials.
The third subject had participated in the prior experiments. Here he discriminated trials with and without targets perfectly in the single-mask condition, but he only discriminated targets with $35 \%$ accuracy. In the dual mask condition, he discriminated targets with $100 \%$ accuracy.

Behaviorally, then, the dual mask condition resulted in a very marked improvement in the accuracy of discriminating targets that were discriminated with approximately chance accuracy in the single mask condition. It might be noted that these behavioral results are much superior to those reported by Dember and Purcell (1967) and Robinson (1966), but the stimulus conditions are also quite different. Our result has since been replicated (Dember, Schwartz, \& Kocak, 1978) in a larger group of subjects.

Figure 5 illustrates the effect of single and dual masks on target residuals for one subject - the results for the other two were quite similar. The residual AERs from both single- and dual-mask conditions show an initial rise from baseline of about $4 \mu \mathrm{V}$; the following positive swing, peak to peak, is about $7 \mu \mathrm{V}$ for the single-mask condition and about $6 \mu \mathrm{V}$ for the dual condition. Thus, the residuals are essentially the same despite markedly different discrimination performances- $35 \%$ vs. $100 \%$.

The subject of Figure 5 participated in all three experiments. The residuals here are nearly identical to those of Experiment 1 (Figure 3) with its different stimuli but similar, monoptic stimulating conditions; they are about half the amplitude of those of Experiment 2 , despite its similar stimuli but dichoptic stimulating conditions. Thus, these data also suggest that dichoptic conditions yield larger residuals than monoptic conditions, i.e., retinal interactions between the stimuli are the major cause of residual response degradation.

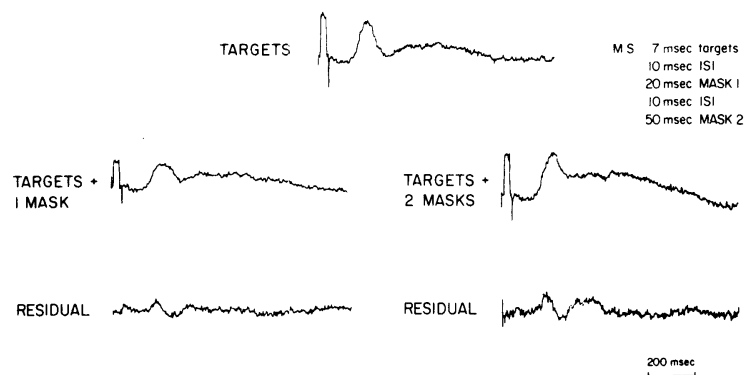

Figure 5. Comparison of single and double mask conditions; monoptic, foveal presentation for one subject. AER to targets alone at the top; second line shows AERs to targets and masks together, single-mask condition on the left, dual-mask condition on the right; third line shows the respective residuals. With one mask, the subject correctly discriminated $35 \%$ of the targets; introduction of a second mask resulted in $100 \%$ correct discrimination of the targets. Despite marked performance differences, the AER residuals are entirely comparable. (All calibration signals are $10 \mu \mathrm{V}$; there is a difference in display size between the singleand dual-mask conditions.) 


\section{GENERAL DISCUSSION}

In three demonstrations with diverse stimulation conditions, we have shown that despite masking of a perceptual discrimination, neural residuals to the target stimuli may still be extracted. Of even greater interest is the fact that when target discrimination was restored, through the use of a second mask, the residual AER was essentially unchanged Since the target residual remained degraded, it can be concluded that accurate target discrimination is not related to total AER amplitude. But "recovered" targets were different from unmasked targets in appearance, suggesting that the difference between AERs to targets presented alone and the residuals obtained here may represent processes which "elaborate" or amplify the essential physical features of the stimuli. Our data imply that evoked responses may be "dissected" into several portions. Some minimal portion apparently reflects activity devoted to detecting the physical characteristics of the eliciting stimuli; this minimum may be necessary, but not sufficient, for subjects to make accurate simple discriminations among stimuli.

Our demonstrations also imply that a major proportion of the reduction in the amplitude of the residuals obtained may be due to interactions between target and mask stimuli at the retina; but masking obtained with patterned stimuli is not dependent on that amplitude reduction, i.e., dichoptic procedures avoid much of the reduction and dichoptic parafoveal procedures must involve masking at cortical, rather than retinal or geniculate, levels.

Finally, it must be noted that our demonstrations all employed target discrimination as the criterion of masking; it remains to be seen if the results obtained are applicable to target detection.

\section{REFERENCES}

Coenen, A. M. L., \& Eunman, E. G. J. Cat optic tract and geniculate unit responses corresponding to human visual making effects. Experimental Brain Research, 1972, 15, 441-451.

Dember, W. N., \& Purcell, D. G. Recovery of masked visual targets by inhibition of the masking stimulus. Science, 1967, 157, 1335-1336.

Dember, W. N., Schwartz, M., \& Kocak, M. Substantial recovery of a masked visual target and its theoretical interpretation. Bulletin of the Psychonomic Society, 1978, 11, 285-287.

Donchin, E., \& Lindsley, D. B. Perceptual masking and enhancement. Electroencephalography and Clinical Neurophysiology, 1965, 19, 325-335.

Donchin, E., Wicke, J. D., \& Lindsley, D. B. Cortical evoked potentials and perception of paired flashes. Science, 1963, 141, $1285-1286$.

Fehmi, L. G., Adxins, J. W., \& Lindsley, D. B. Electrophysiological correlates of visual perceptual masking in monkeys. Experimental Brain Research, 1969, 7, 299-316.

Robinson, D. H. Disinhibition of visually masked stimuli. Science, 1966, 154, 157-158.

SCHILlER, P. H. Behavioral and electrophysiological studies of visual masking. In $\mathrm{K}$. N. Leibovic (Ed.), Information processing in the nervous system. N.Y: Springer-Verlag, 1969.

SChiller, P. H., \& ChOROVER, S. L. Metacontrast: Its relation to evoked potentials. Science, 1966, 153, 1398-1400.

Schwartz, M., \& Shagass, C. Recovery functions of human somatosensory and visual evoked potentials. In R. Katzman (Ed.), Sensory evoked response in man. Annals of the New York Academy of Sciences, 1964, 112, Art. 1, 510-525.

TuRvey, M. T. On peripheral and central processes in vision: Inferences from an information-processing analysis of masking with patterned stimuli. Psychological Review, 1973, 80, 1-52.

Vaughan, H. G., JR., \& Silverstein, L. Metacontrast and evoked potentials: A reappraisal. Science, 1968, 160, 207-208.

(Received for publication May 3, 1978; revision accepted January 4, 1979.) 